\title{
Explaining the formation of bulges with MOND
}

Françoise Combes

\begin{abstract}
In the cold dark matter (CDM) paradigm, bulges easily form through galaxy mergers, either major or minor, or through clumpy disks in the early universe, where clumps are driven to the center by dynamical friction. Also pseudo-bulges, with a more disky morphology and kinematics, can form more slowly through secular evolution of a bar, where resonant stars are elevated out of the plane, in a peanut/box shape. As a result, in CDM cosmological simulations, it is very difficult to find a bulgeless galaxy, while they are observed very frequently in the local universe. A different picture emerges in alternative models of the missing mass problem. In MOND (MOdified Newtonian Dynamics), galaxy mergers are much less frequent, since the absence of dark matter halos reduces the dynamical friction between two galaxies. Also, while clumpy galaxies lead to rapid classical bulge formation in CDM, the inefficient dynamical friction with MOND in the early-universe galaxies prevents the clumps to coalesce together in the center to form spheroids. This leads to less frequent and less massive classical bulges. Bars in MOND are more frequent and stronger, and have a more constant pattern speed, which modifies significantly the pseudo-bulge morphology. The fraction of pseudo-bulges is expected to be dominant in MOND.
\end{abstract}

\section{Introduction}

Although the standard CDM model for dark matter is the best frame to represent the universe at large scales, and account for galaxy formation, it experiences difficulties at galaxy scale (e.g. Moore et al. 1999, Silk \& Mamon 2012). Cosmological simulations in the standard model predict an over concentration of dark matter in galaxies, and cuspy density profiles, instead of

Observatoire de Paris, LERMA, CNRS, 61 Av. de l'Observatoire, F-75014, Paris, France e-mail: francoise.combes@obspm.fr 
the density cores derived from rotation curves, especially in low-mass galaxies (e.g. de Blok et al 2008, Swaters et al 2009). Also simulations have difficulties to form large galaxy disks, since the angular momentum of baryons is lost against massive dark halos (e.g. Navarro \& Steinmetz 2000), and the missing satellites problem remains unsolved (Diemand et al. 2008). In addition, observed low-mass satellites of the Milky Way have a much larger baryonic fraction than expected from halo abundance matching (e.g. Boylan-Kolchin et al. 2011, 2012).

A large numerical effort has been spent to solve these problems by the detailed physics of the baryonic component, in particular star formation and AGN feedback (e.g. Vogelsberger et al. 2014, Schaye et al. 2015). Another track is to explore alternatives to dark matter models, and in particular modified gravity scenarios, able to account for the missing mass in galaxies.

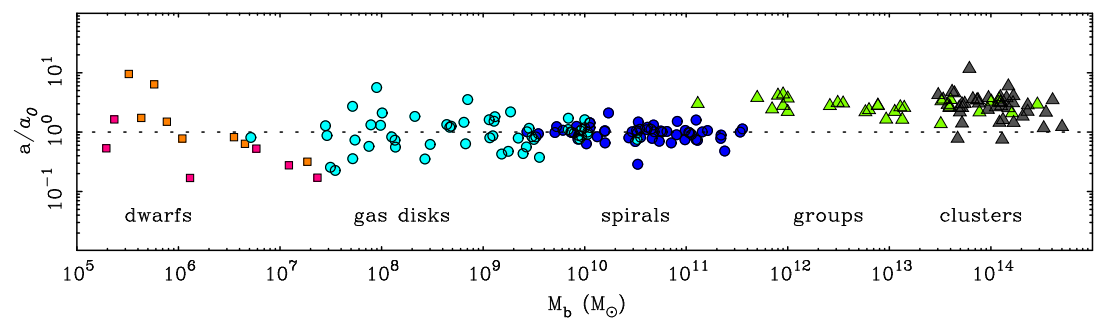

Fig. 1 The observed parameter $\mathrm{V}_{f}^{4} /\left(\mathrm{GM}_{b}\right)$, where $\mathrm{V}_{f}$ is the rotational velocity taken in the flat portion of the rotation curve, and $\mathrm{M}_{b}$ is the baryonic mass, can be also written as the acceleration of the system: $\mathrm{a}=\mathrm{V}_{f}^{2} / \mathrm{R}$, and $\mathrm{R}=\mathrm{GM} / \mathrm{V}_{f}^{2}$. The very small deviation of a from the constant $\mathrm{a}_{0}$ is remarkable, given the large range of ten decades in baryonic mass $\mathrm{M}_{b}$. This observation is somewhat puzzling for the standard dark matter model, but is the basis of the modified gravity (MOND) model (from Famaey \& McGaugh 2012).

Already 30 years ago, Milgrom (1983) had the idea of the MOdified Newtonian Dynamics (MOND), based on the fundamental observation that the missing mass problem occurs only in the weak field regime, at low acceleration, when $a$ is lower than the characteristic value of $\mathrm{a}_{0}=210^{-10} \mathrm{~m} / \mathrm{s}^{2}$. The observed flat rotation curves in the outer parts of galaxies suggests that in this regime the actual acceleration varies in $1 / \mathrm{r}$. Galaxies are also following the baryonic Tully-Fisher relation (McGaugh et al. 2000), where the baryonic mass of a system is proportional to the 4 th power of the maximum rotational velocity (see Figure 1). Milgrom then proposes that at acceleration below $\mathrm{a}_{0}=210^{-10} \mathrm{~m} / \mathrm{s}^{2}$, the gravitational attraction will tend to the formulation a $=\left(\mathrm{a}_{0} \mathrm{a}_{N}\right)^{1 / 2}$, where $\mathrm{a}_{N}$ is the Newtonian value. This effectively produces an acceleration in $1 / \mathrm{r}$, implying a flat rotation curve in the limiting regime, and leading automatically to the Tully-Fisher relation. The transition between the Newtonian and MOND regime is controlled by an interpolation function $\mu(\mathrm{x})$, of $\mathrm{x}=\mathrm{a} / \mathrm{a}_{0}$, which standard form is $\mu(\mathrm{x})=\mathrm{x} /\left(1+\mathrm{x}^{2}\right)^{1 / 2}$. It essentially tends to $\mathrm{x}$ in the MOND regime, when $\mathrm{x}$ is smaller than 1 , and to unity in 
the Newtonian regime. This phenomenology has a large success explaining rotation curves and kinematics of galaxies, from dwarf irregulars dominated by dark matter (and therefore in the MOND regime), to the giant spirals and ellipticals, dominated by baryons (e.g. Sanders \& McGaugh 2002). Although the model is still empirical, it is possible to build relativistically covariant theories, able to reproduce gravitational lensing and other phenomena, while tending asymptotically to the above formulation in the non-relativistic limit (Bekenstein 2004).

The galaxy dynamics is quite different in the MOND hypothesis with respect to the standard dark matter model. Some phenomena have already been explored (see e.g. the review by Famaey \& McGaugh 2012), but many are still to be discovered, and in particular galaxy formation, and high redshift evolution. The stability of galaxy disks is fundamentally different, provided that they have low surface brightness (LSB), and are close to the MOND regime (Milgrom \& Sanders 2007). Since the MOND disks are completely self-gravitating, they could be much more unstable, however the acceleration is varying asymptotically as the square root of the mass (and not linearly with the mass), so the final effects are not intuitive. Bars are forming quickly in MOND disks, and their pattern speed is not declining through dynamical friciton against a dark matter halo, so resonances are long-lived, and may have more impact (Tiret \& Combes 2007). Galaxy interactions with no extended dark halos suffer much less dynamical friction, and mergers are rare (Tiret \& Combes 2008b). This changes very significantly the hierachical scenario of galaxy formation, and in particular bulge formation. Therefore, although bulges are now generally in the Newtonian regime today, their formation is certainly very different in the MOND frame with respect to the standard model. Bulges are increasingly important along the Hubble sequence towards the early-types, which correspond to the more massive end. For giant galaxies, the low acceleration regime is encountered only in the outer parts, and the central parts remain Newtonian. Only dwarf galaxies and LSB objects without bulges are still in the MOND regime in their center. This means that bulges today are not likely to be affected by a modified dynamics.

In the following, we will consider in turn the main dynamical mechanisms to form bulges in the $\Lambda \mathrm{CDM}$ paradigm:

- Mergers, major or a series of minor mergers

- Secular evolution, bars and the formation of pseudo-bulges

- Clumpy galaxies at high redshift and dynamical friction

Are all these processes also at work in MOND, and with which efficiency? It is well known that the standard $\Lambda \mathrm{CDM}$ model has difficulties to account for the large number of observed bulge-less galaxies (Kormendy et al. 2010). Is this problem solved by MOND? 


\section{Galaxy mergers}

In the standard hierarchical scenario, galaxy mergers play a large role in mass assembly, and one of the results of the repeated coalescence of galaxies is to randomly average out the angular momentum of the system, and to form spheroids (e.g. Toomre 1977, Barnes \& Hernquist 1991, Naab \& Burkert 2003, Bournaud et al. 2005, 2007a). In these last works, it was shown how repeated minor mergers progressively accumulate stars in a central spheroid and grow the bulge, to transform the galaxy in a more early-type spiral. Eventually, N minor mergers of mass ratio N:1 result in an elliptical remnant quite similar to those formed in a 1:1 merger. As shown by Barnes (1988), mergers are very efficient in forming long tidal tails while the main baryonic components merge quickly, because of the existence of extended and massive dark halos, which take the orbital angular momentum away. It can then be expected that the frequency of mergers will depend crucially on the model assumed for the missing mass.

\subsection{Major mergers in MOND}

One of the main questions is to know whether the MOND dynamics is able to produce long tails in major mergers of galaxies, like in the prototypical Antennae system (Figure 2). These tails have also helped to constrain the dark matter halos potential (Dubinski et al. 1996). With MOND, the result is not easy to predict, and numerical simulations are necessary, since the External Field Effect (EFE) perturbs the MONDian dynamics in the outer parts of galaxies. This new effect particular to MOND comes from the fact that it violates the Strong Equivalence Principle of General Relativity. In the Newtonian frame, the internal gravitational forces of a system are independent of their external environment: if the object is embedded in a large system, exerting a force which can be considered constant all over the object, then the internal dynamics is unchanged. Of course, if the force is varying across the object, its differential gives rise to tidal forces, which impact the object. But in the MOND dynamics, even a constant force may create an acceleration above the critical one $a_{0}$, and get the object out of the MOND regime (Milgrom 1983, 1998).

Several cases can be distinguished to model the EFE, according to the respective values of the external acceleration $\mathrm{a}_{e}$ with respect to the internal acceleration a of the object under consideration, and the critical acceleration $\mathrm{a}_{0}$. If $\mathrm{a}_{e}<\mathrm{a}<\mathrm{a}_{0}$, then the standard MOND effects are retrieved, and if $\mathrm{a}<\mathrm{a}_{0}<\mathrm{a}_{e}$, then the EFE is strong enough to make the system purely Newtonian. But in the intermediate regime, where $\mathrm{a}<\mathrm{a}_{e}<\mathrm{a}_{0}$ then the system is Newtonian with a renormalised gravitational constant G. It can be estimated for instance in a one-dimensional system, that the effective 
gravitational constant is then $\mathrm{G}_{e f f}=\mathrm{G}\left[\mu_{e}\left(1+\mathrm{L}_{e}\right)\right]^{-1}$ where $\mu_{e}=\mu\left(\mathrm{a}_{e} / \mathrm{a}_{0}\right)$ and $\mathrm{L}_{e}$ is the logarithmic gradient of $\mu$ (Famaey \& McGaugh 2012). In the outer parts of a given system, the internal acceleration is always vanishing, and there will always be a small $\mathrm{a}_{e}<\mathrm{a}_{0}$, therefore this represents the general case: the gravitational force falls again as $1 / \mathrm{r}^{2}$, and the potential as $1 / \mathrm{r}$ and not logarithmically, as could be extrapolated. This allows to define the escape velocity of the system, as in the Newtonian case. Computations of the EFE in the Milky Way, due to the nearby Andromeda galaxy, have given results compatible with the observations (Wu et al. 2007).

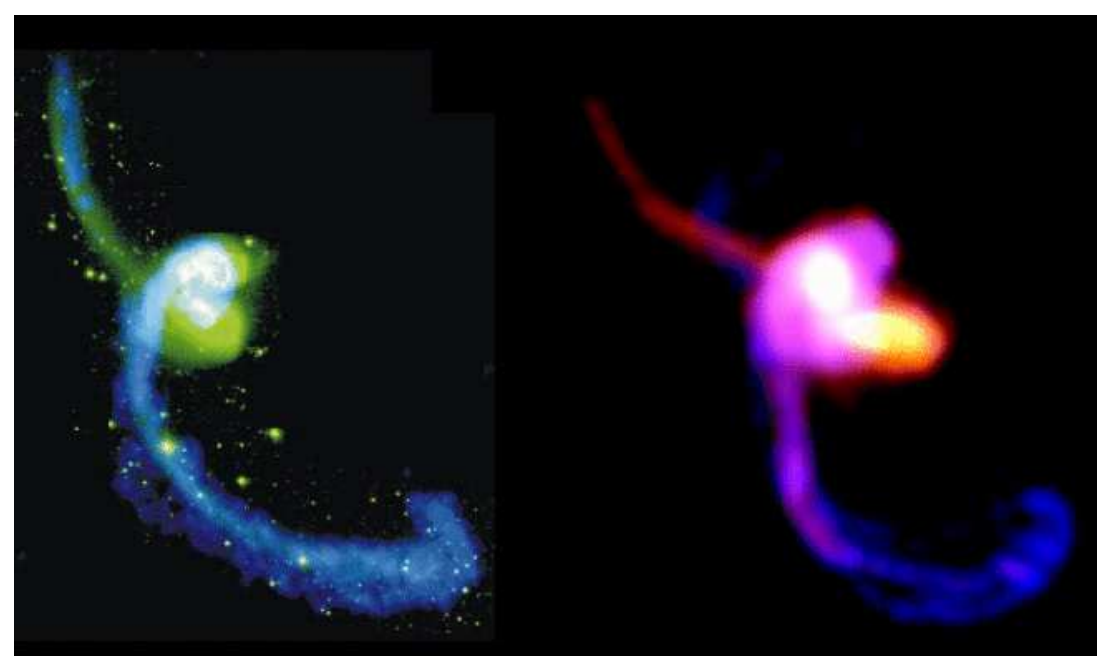

Fig. 2 Simulation of tidal interactions in a major merger in MOND (right, with gas in blue and stars in red) compared to the Antennae galaxies (Hibbard et al. 2001, HI gas in blue, and stars in green). The two long tidal tails are reproduced (Tiret \& Combes 2008b).

Simulations with a 3D adaptive-mesh code able to solve the MOND equations, and including gas and stars, have shown that two long tidal tails can develop in a major merger similar to the Antennae (cf Figure 2). In absence of dark matter particles as receivers of the orbital angular momentum of the two galaxies, baryons are playing this role, and tidal tails can be very long. In addition, tidal dwarf galaxies can be naturally formed at the tip of the tidal tails in MOND, while it requires radially extended dark matter halos in the standard model (Bournaud et al. 2003). The big difference between the two models is the efficiency of the dynamical friction. While mergers can take only one orbit, or less than one Gyr in the standard model, it will take several Gyrs with MOND, and mergers will occur only with selected impact parameters, and initial relative angular momenta. At a distance of $\sim 100 \mathrm{kpc}$, two galaxies in circular orbits will not merge in a Hubble time with MOND (Figure 3). On the contrary, in the standard model, galaxies have already 
plunged well inside their dark matter halos, of radius $\sim 200 \mathrm{kpc}$. Then local dynamical friction is already effective, while in the MOND case, the relative decay relies only on the friction at distance, which is much weaker.

\subsection{Dynamical friction}

As described above, the gravitational forces between galaxies at large distance are likely to vary as $1 / \mathrm{r}^{2}$ as in the Newtonian regime, but with a boosted constant, so the long-distance approach of galaxies could be thought similar. However the phenomena associated to dynamical friction are completely different. Answers to this problem have been controversial at the start, since Ciotti \& Binney (2004) computed the relaxation time in the MOND regime with strong approximations: very small fluctuations, impulse approximation for deflection or orbits, linear summation of effects, etc. and they compare this two body relaxation time with that in the Newtonian regime, considering the dark matter halo as a rigid background, not participating in the fluctuations. Then they extrapolate their finding of a shorter relaxation time in MOND to the dynamical friction time, obtained for test particles for the local formula of Chandrasekhar (1943), and conclude that globular clusters should spiral inwards to the center in dwarf galaxies in a few dynamical timescales, as well as galaxies in groups and in clusters. Nipoti et al. (2008) tried to confirm these findings in simulations, by applying the same hypotheses of a tiny perturbation: the massive bodies subject to the friction, either globular clusters or a rigid bar, have to contain less than $5 \%$ of the baryonic mass, so that particles absorbing the energy and angular momentum are not globally perturbed. In realistic systems though, Nipoti et al. (2007) found that the merging timescales for spherical systems are significantly longer in MOND than in Newtonian gravity with dark matter, and Tiret \& Combes (2007) found that bars keep their pattern speed constant in MOND, while they are strongly slowed down in the Newtonian equivalent system with a dark matter halo. In summary, dynamical friction is very slow in MOND, since galaxies are not embedded in extended and massive spheroids of dark matter particles, able to accept the orbital angular momentum. A short merging time-scale for equal-mass interacting galaxies, as short as the CDM, is possible only for nearly radial orbits. Although the impact of very small fluctuations could be larger in MOND than in Newtonian dynamics, the effect saturates quickly when the perturbation is no longer infinitesimal, and on the contrary the equivalent Newtonian system with dark matter has shorter response timescales, and a massive body (either a companion, or a galactic bar) is slowed

down very efficiently. The case of bars, their pattern speeds, and their impact on bulge formation will be detailed in the next Section 3 , 


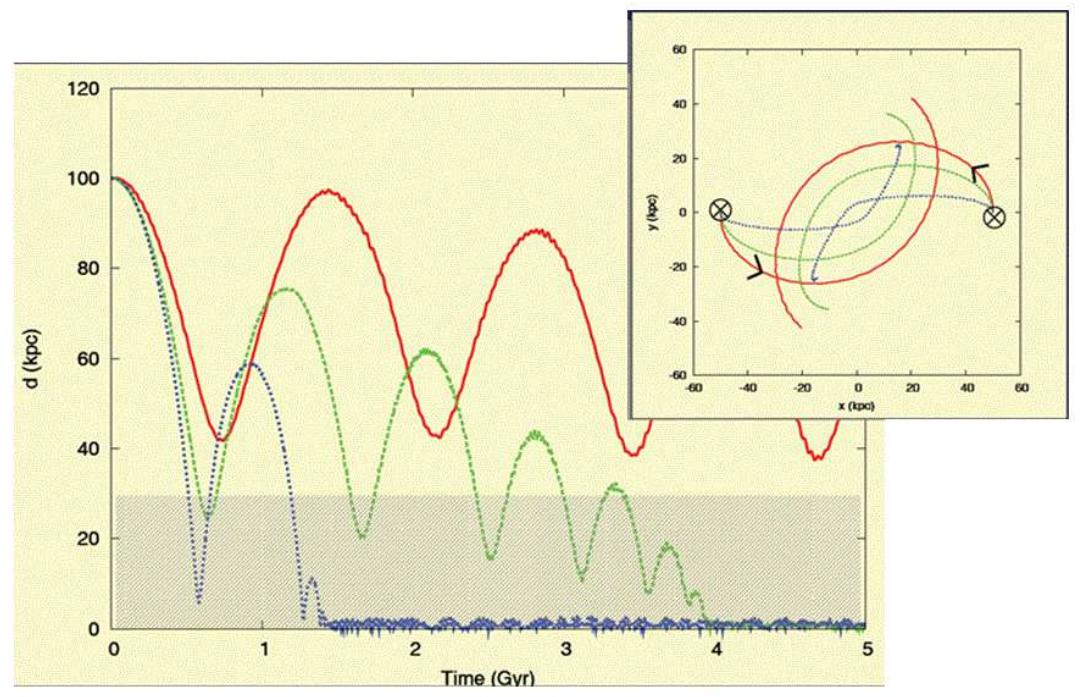

Fig. 3 Radial decay during the tidal interactions between two equal-mass spiral galaxies, in the MOND model (Tiret \& Combes 2008b). At left is shown the relative distance in kpc versus time in Gyr, while the insert at right shows the corresponding trajectories. (image by $\mathrm{O}$. Tiret).

Could the smaller merger frequency predicted by MOND be tested in observations? Unfortunately, the actual merger frequency is not directly accessible. Observers tend to quote galaxy pair frequency, or starbursts due to mergers (e.g. Bell et al. 2006, Lopez-Sanjuan et al. 2013, Stott et al. 2013). However, there is a degeneracy here, since galaxy can appear in pairs during either a short or long time-scale, and starbursts can occur at each closer passage. In the standard DM models, an assumption is done on the duration of galaxy interactions, and the number of starbursts: according to the initial relative velocity and the geometry of the encounter, the merger is expected to occur in one or two passages. An intense starburst is associated to the final phase, and the number of starbursts is thought to count the number of mergers (e.g. Di Matteo et al 2007). In the MOND model, many passages in binary galaxies will be required before the final merging, and a starburst may be triggered at each pericenter. The number of starbursts as a function of redshift could then be similar, and cannot discriminate the two models. The degeneracy cannot be raised between a limited number of long-lived mergers, or a high frequency of short-lived mergers. 


\section{Bars}

To probe realistically the stability of disks with the MOND dynamics, numerical simulations have been run, solving the N-body problem on a grid, through the equations of Bekenstein \& Milgrom (1984). Brada \& Milgrom (1999) showed that disks were always more instable in MOND. For the equivalent Newtonian system with a spherical dark halo, the more unstable galaxies are those with massive disks, which are more self-gravitating, while low-mass disks are stabilized by their halo. In MOND, the instability is about the same for massive disks, which are still in the Newtonian regime, however, low-mass disks remain unstable, and their growth rate tend to a constant, instead of vanishing.

\subsection{Disk stability in MOND}

From detailed comparison of two identical initial disks simulated with Newtonian dynamics+dark matter and MOND, Tiret \& Combes (2007) have shown that bars develop quicker with modified gravity (see Figure 4). To have identical starts, the baryonic disk is first computed in equilibrium with its velocity distribution in MOND, and then, the amount of dark matter required to obtain the same derived rotation curve, is added for the Newtonian dynamics run. The evolution of the bar strength in Figure 4 reveals that both bars experience a drop in their strength, and this is due to the vertical resonance, building a peanut-shape feature, evolving in a pseudo-bulge (e.g. Combes \& Sanders 1981, Combes et al. 1990, Bureau \& Freeman 1999). The peanut occurs later in MOND. The bar remains strong during a longer time-scale, but then weakens, while the Newtonian bar can strengthen again, by exchanging angular momentum with the dark halo (e.g. Athanassoula 2002).

This different way of growing results also in a different final morphology of the stellar disks: in MOND the disk is more extended, since the bar has grown by angular momentum exchange with the outer disk particules. Figure 4 represents an early-type spiral Sa. When all types are considered, the bar occurs much later in Newtonian models, because later types are more dominated by the dark matter halo, and are less self-gravitating. In MOND it is the contrary, the bar is first stronger in late-types, and then the disk is heated too much and the bar weakens. When the statistics are computed over the whole Hubble sequence, it appears that bars are stronger and more frequent in MOND, when only stellar components are taken into account. The higher MOND bar frequency is more in agreement with observations, where $2 / 3 \mathrm{rds}$ of spiral galaxies are barred (e.g. Laurikainen et al. 2004, 2009). 

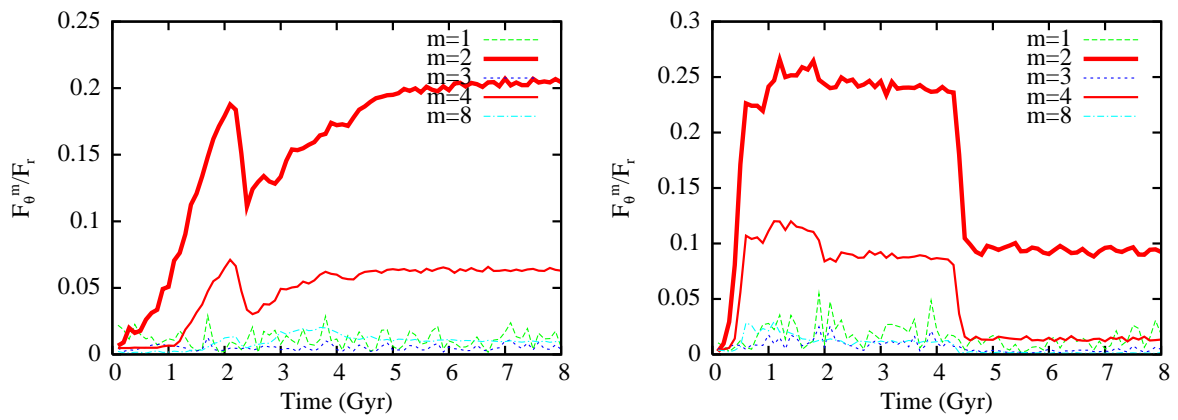

Fig. 4 Strength of the bar formed in an Sa-type galaxy purely stellar simulation, measured by its Fourier harmonics $m=2,3,4$ and 8 (ratio of tangential to radial force), for the CDMNewton model (left) and MOND (right). The bar settles earlier in MOND, and stays longer, but after dropping at $4.5 \mathrm{Gyr}$, it does not develop again as in the CDM (cf Tiret \& Combes 2007). The drop at 2.5 Gyr in the DM model as in the MOND model at 4.5 Gyr is due to the formation of a peanut bulge, through the vertical resonance (e.g. Combes et al. 1990).

\subsection{Pattern speed evolution}

The bar pattern speed evolutions are also different in the two models. As shown in Figure 5 left, $\Omega_{\text {bar }}$ is almost constant in MOND, while it drops by a factor 3 in 7 Gyr time in the equivalent Newtonian system. This is clearly due to the exchange of angular momentum from the bar to the dark matter halo, through dynamical friction. Indeed, the test run when the Newtonian system is computed with a rigid halo, which cannot deform and produce dynamical friction, has an almost constant $\Omega_{b a r}$ too.
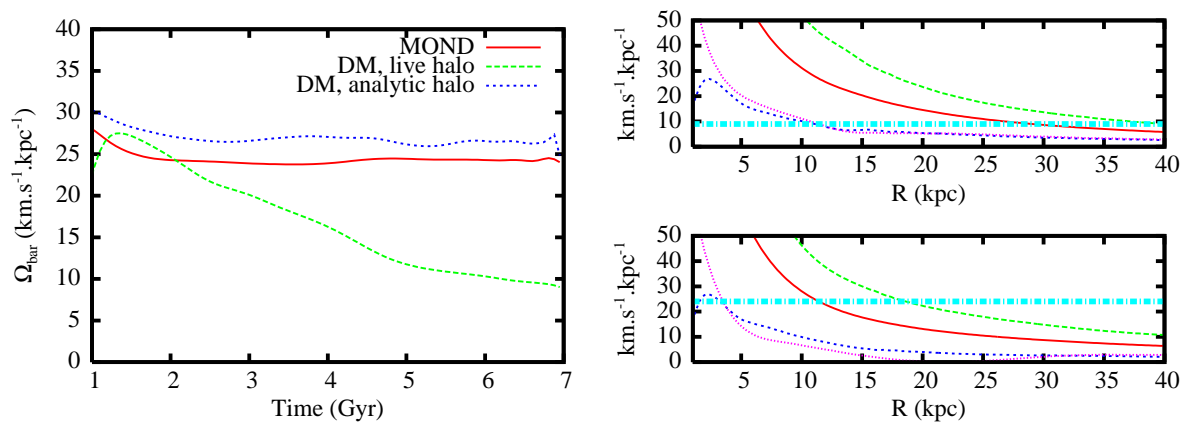

Fig. 5 Left Bar pattern speeds versus time: in MOND, the pattern speed remains constant, as in the Newtonian galaxy with a rigid dark matter halo. When the dark halo particles are taken into account self-consistently, the bar slows down, losing its angular momentum through dynamical friction. Right Frequency curves (from bottom to top, $\Omega-\kappa / 2, \Omega-\nu_{z} / 2, \Omega$ and $\Omega+\kappa / 2$ ) for the CDM case (top) and MOND (bottom). The thick horizontal line is the pattern speed of the bar in each case (cf Tiret \& Combes 2007). 
This drop in $\Omega_{\text {bar }}$ for the Newtonian+dark matter model has several consequences: First the Lindblad resonances in the plane and the vertical resonance move in radius, as shown in Figure 5 right. The pattern speed at the end of the simulation is shown as a thick dash line, and the inner/vertical resonance moves from $2 \mathrm{kpc}$ to $12 \mathrm{kpc}$. Since the peanut represents stars vertically up-lifted at resonance, this means that the radius of the peanut is moving radially outwards, as shown in Figure 6. In MOND on the contrary, resonances are more long-lived, and can produce more robust effects.

\subsection{Bulges and pseudo-bulges}

Until now, the comparison between MOND and the Newtonian equivalent systems has been discussed with purely stellar disks. However, the presence of gas, and its interaction with stars change the picture. Gas as a dissipational component, is subject to a phase shift in its response to the bar pattern. There is a torque from the bar to the gas, that drives it to the center. This changes the potential there, and therefore the $\Omega$ frequencies and the resonances. The final result is a weakening of the bar, which can only develop again through gas accretion (e.g. Bournaud \& Combes 2002). Gas dissipation and star formation have been taken into account in MOND simulations by Tiret \& Combes (2008a). Statistically, bars occur even more rapidly in gas rich disks, and especially in the Newtonian models, which were too stable in the purely stellar disks. This makes the two models more similar, as far as the frequency of bars is concerned. Since the baryonic mass is more concentrated with gas in any model, the vertical resonance and the peanut occur at smaller radii, therefore the pseudo-bulges are smaller and more boxy in appearance.

Finally, the gas is driven by gravity torques inwards inside corotation, and outwards outside. It accumulates in rings at the inner (outer) Lindblad resonances respectively, in star forming rings that reproduce the blue rings observed in barred galaxies (e.g. Buta \& Combes, 1996). In MOND, this phenomenon is even more remarkable, since first bars are still stronger and more frequent than in the Newtonian dynamics, but also the exchange of angular momentum between the stellar and the gas components is favored, while in the Newtonian case, there is competition with the dark halo for this exchange.

Summarizing the previous learnings, bars are more frequent in MOND, and consequently the formation of pseudo-bulges is favored. The fraction of classical bulges formed in major or minor mergers is likely to be much less, so that the picture of bulge formation is significantly different in the two regimes. These conclusions are applicable mainly to the local galaxies, at very low redshifts. First bars are less frequent in the past (Sheth et al. 2008), and pseudo-bulges are thought to be the dominant bulge formation at lower redshift (e.g. Kormendy \& Kennicutt 2004). Second, it is not well known how 

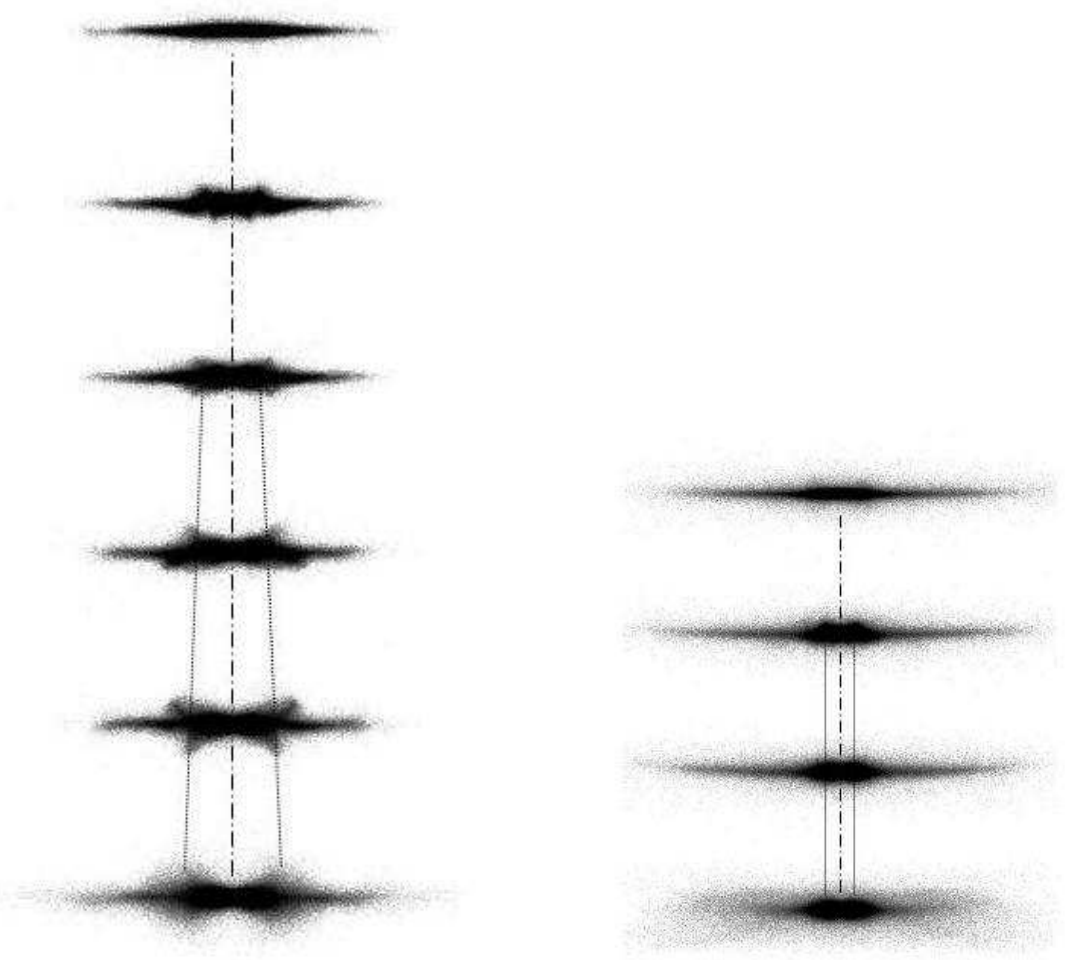

Fig. 6 Peanut-shape bulge formation, through vertical resonance with the bar. With CDM (left), the bar slows down with time, and the resonance moves to larger radii. Two peanut features are formed along the evolution, and the last one is rather extended in radius, while with MOND (right), there is only one peanut formed, centrally concentrated (Tiret \& Combes 2007). These runs consider only the stellar component. Peanuts are less developed, when the disk is rich in gas.

the MOND model can be extended at high redshift. It has been remarked that the critical acceleration $\mathrm{a}_{0}$ is of the same order as $\mathrm{c} \mathrm{H}_{0}$, with $\mathrm{H}_{0}$ the Hubble constant today, and therefore the critical acceleration could increase with $\mathrm{z}$ as $\mathrm{H}(\mathrm{z})$. Similarly $\mathrm{a}_{0} \sim \mathrm{c}(\Lambda / 3)^{1 / 2}$ (with $\Lambda$ being the dark energy parameter), and any kind of variation with time of $\mathrm{a}_{0}$ is possible.

In the standard model, there is another mechanism to form bulges, which is more dominant at high redshift, that we will consider now. 


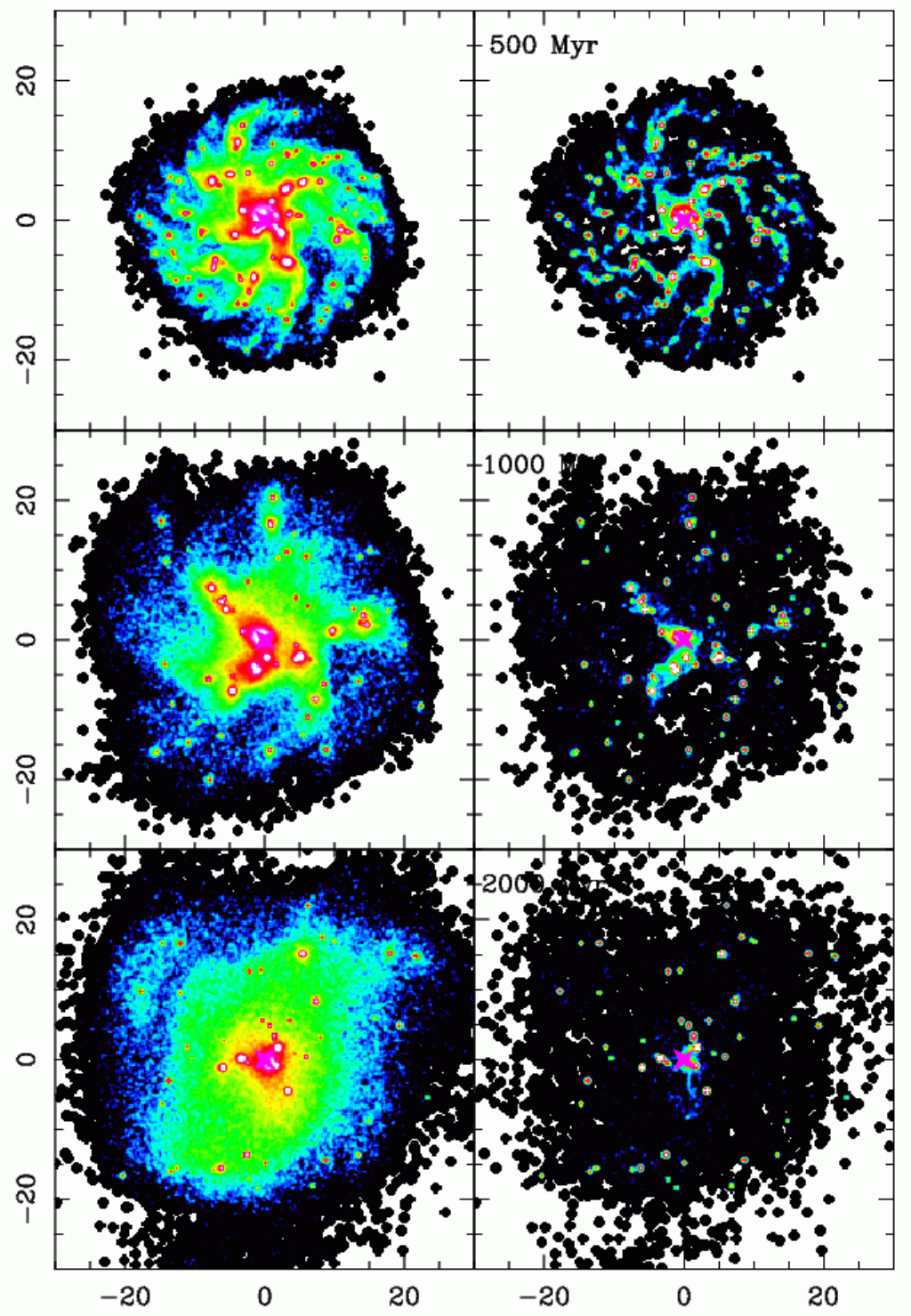

Fig. 7 All baryons (left) and gas (right) surface densities of the dwarf clumpy galaxy, simulated with MOND gravity, at epochs 0.5, 1 and 2 Gyr. Each panel is $60 \mathrm{kpc}$ in size. The color scale is logarithmic and the same for all plots. From Combes (2014).

\section{Clumpy disks}

When the universe was about half of its age ( $\mathrm{z} \sim 0.7)$ and earlier, the morphology of spiral galaxies were significantly different from what we know today, in 
the Hubble sequence. Galaxies were much more clumpy, with clumps of gas and stars of kpc size (e.g. Elmegreen 2007). These very irregular morphologies are thought to result from the very high gas fraction of these early galaxies. Noguchi (1999) simulated the formation of galaxies from highly gaseous systems, and found that they form giant clumps, which by dynamical friction can spiral inwards to the center rather quickly to form a bulge. Bournaud et al (2007b) developed further the dynamical mechanisms, and showed that rather quickly, clumpy disks form an exponential disk, a bulge, and also a thick disk due to the stars formed in the turbulently thick gaseous disk. The disruption of the clumps by the feedback of star formation (supernovae, winds) is not yet well known, and can be adjusted to maintain the clumpy disks at the observed frequency (Elmegreen et al. 2008) The large increase of the gas fraction of spiral galaxies with redshift has been confirmed by direct observations of the molecular gas (e.g. Tacconi et al. 2010).

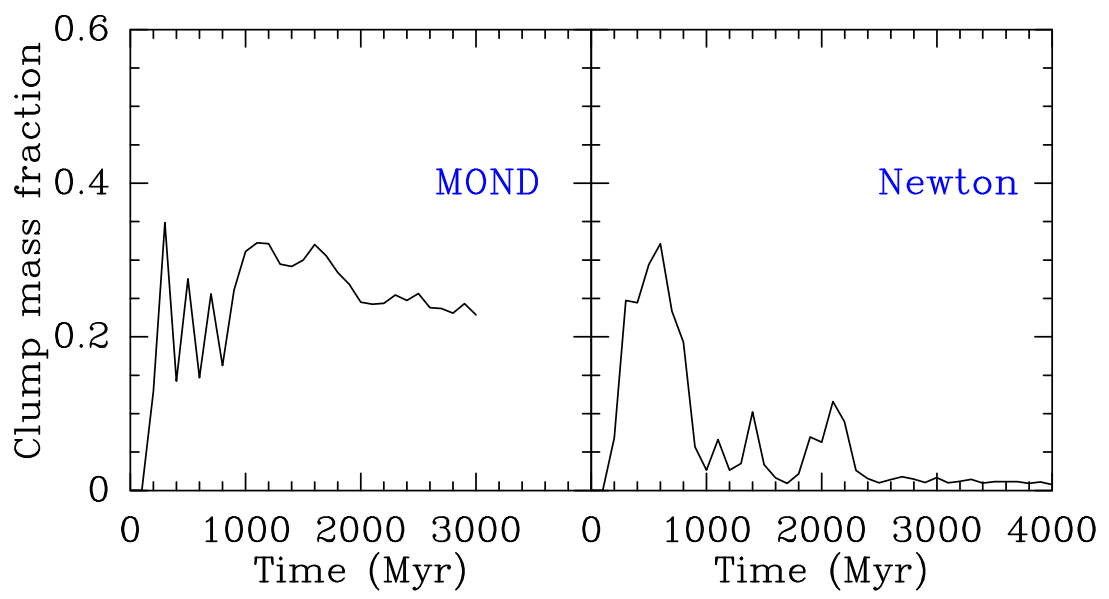

Fig. 8 Evolution of the clump mass fraction for the giant galaxy, in the MOND gravity (left) and in the Newtonian gravity (right).

The very high efficiency of bulge formation through dynamical friction in clumpy disks might be a problem for the standard dark matter model, since bulge-less galaxies are quite frequent today (e.g. Weinzirl et al. 2009). Since dynamical friction occurs mainly against dark matter halos, it is expected that it will be much less important in the MOND dynamics, and the rapid bulge formation could be avoided. This was indeed demontrated in a recent paper, comparing formation of bulges in gas-rich clumpy galaxies, in the two gravity models, Newtonian with dark matter and MOND (Combes, 2014).

This work first computes the dynamical time-scale in an idealized situation, where the galaxy disks are purely stellar, to isolate the main dynamical phenomenon, from the more complex gas hydrodynamics, star formation or 
feedback. When several clumps are launched randomly in the disk, the dynamical friction efficiency is difficult to predict, since the wakes of the different massive bodies interfere (Weinberg 1989). With typical clump mass fraction (25-30\%), in the Newtonian model, the dynamical time-scale for clumps to spiral into the center of a galaxy with baryonic mass $610^{10} \mathrm{M}_{\odot}$ is $0.3 \mathrm{Gyr}$, and $1 \mathrm{Gyr}$ for a galaxy with baryonic mass $610^{9} \mathrm{M}_{\odot}$. In the MOND regime, the clumps do not fall into the center before 3 Gyr. When the gas and star formation/feedback are taken into account, the simulated galaxy disks are rapidly unstable to clump formation, due to the gas fraction of $50 \%$. In the Newtonian gravity with dark matter, previous results are retrieved, i.e. an increasing clump mass fraction in the first $200 \mathrm{Myr}$, and the coalescence of clumps towards the center, with a spheroidal bulge formation, in less than 1 Gyr (Noguchi 1999, Immeli et al 2004, Bournaud et al. 2007b). With MOND gravity, clumps form quickly too (cf Fig 7), but they maintain in the disk for the whole simulation of $3 \mathrm{Gyr}$, until the gas has been consumed in stars. The clump mass fraction does not decrease much, being just eroded through stellar feedback, and shear forces (Fig. 8). Bulges are clearly not formed in the early clumpy phase of galaxy formation, as in the Newtonian equivalent systems.

\section{Conclusions}

In the standard model, classical bulges are thought to be formed essentially in galaxy mergers, which are very frequent in the hierarchical scenario of galaxy formation. In addition, a small classical bulge is also formed in the first Gyr of the galaxy lifes, during the clumpy phase, where their disk is gas dominated. Later on, pseudo-bulges formed out of bar resonances are adding their contribution to the classical bulges.

In the frame of MOND, bulges are hardly formed in early times, in the clumpy phase of galaxy formation, since the dynamical friction without dark matter halos is not efficient enough to drive clumps towards the center, before they are destroyed or reduced by stellar feedback and shear forces. Classical bulges can form later, through hierarchical merging, with a frequency which is smaller than what occurs in the analogous Newtonian systems with dark matter. They however form with comparable frequency through secular evolution, by vertical resonances with bars. It is therefore expected that the contribution of pseudo-bulges with respect to classical bulges is higher in MOND. Globally, bulges are expected less frequent and less massive, which might be more compatible with observations of local galaxies (Weinzirl et al. 2009, Kormendy et al. 2010). These tendencies have to be confirmed with more simulations. A complete cosmological context is however not yet possible, given the uncertainties of the modified gravity models in the early universe. 


\section{References}

Athanassoula E.: 2002 ApJ 569, L83

Barnes, J. E.: 1988, ApJ 331, 699

Barnes, J. E., Hernquist, L. E. 1991, ApJ 370, L65

Bekenstein J., Milgrom M.: 1984, ApJ 286, 7

Bekenstein J.: 2004 PhRvD 70h3509

Bell E.F., Phleps, S., Somerville, R.S. et al.: 2006, ApJ 652, 270

Bournaud F., Combes F.: 2002, A\&A 392, 83

Bournaud F., Duc P.-A., Masset F.: 2003, A\&A 411, 469

Bournaud F., Jog C.J., Combes F.: 2005, A\&A 437, 69

Bournaud F., Jog C.J., Combes F.: 2007a, A\&A 476, 1179

Bournaud F., Elmegreen B.G., Elmegreen D.M.: 2007b ApJ 670, 237

Boylan-Kolchin, M., Bullock, J. S., Kaplinghat, M.: 2011, MNRAS 415, L40

Boylan-Kolchin, M., Bullock, J. S., Kaplinghat, M.: 2012, MNRAS 422, 1203

Brada R., Milgrom M.: 1999, ApJ 519, 590

Bureau, M., Freeman, K. C: 1999, AJ 118, 126

Buta R., Combes F.: 1996, F F $^{\text {als }}$ of Cosmic Physics, Volume 17, pp. 95-281

Chandrasekhar S., 1943, ApJ 97, 255

Ciotti L., Binney J.: 2004, MNRAS 351, 285

Combes F., Sanders R. H.: 1981, A\&A 96, 164

Combes F., Debbasch F., Friedli D., Pfenniger D.: 1990, A\&A 233, 82

Combes F.: 2014, A\&A, 571, A82

de Blok, W. J. G., Walter, F., Brinks, E.. et al.: 2008, AJ 136, 2648

Diemand, J., Kuhlen, M., Madau, P. et al.: 2008, Nature 454, 735

Di Matteo P., Combes F., Melchior A-L, Semelin B.: 2007, A\&A 468, 61

Dubinski J., Mihos J. C., Hernquist L., 1996, ApJ 462, 576

Elmegreen D.M.: 2007, in IAU S235, ed. F. Combes \& J. Palous, CUP, p. 376

Elmegreen B.G., Bournaud F., Elmegreen D.M.: 2008, ApJ 688, 67

Famaey B., McGaugh S. S.: 2012, Living Reviews in Relativity, vol. 15, no. 10

Hibbard J.E., van der Hulst J.M., Barnes J.E., Rich R.M.: 2001, AJ 122, 2969

Immeli A., Samland M., Gerhard O., Westera P.: 2004, A\&A 413, 547

Kormendy, J., Kennicutt, R. C.: 2004, ARAA 42, 603

Kormendy J., Drory N., Bender R., Cornell M. E., 2010, ApJ 723, 54

Laurikainen, E., Salo, H., Buta, R., Vasylyev, S.: 2004, MNRAS 355, 1251

Laurikainen, E., Salo, H., Buta, R., Knapen, J. H.; 2009, ApJ 692, L34

Lopez-Sanjuan, C., Le Fèvre, O., Tasca, L.A.M. et al.: 2013, A\&A 553, A78

McGaugh S.S., Schombert J.M., Bothun G.D., de Blok W.J.G.: 2000, ApJ 533, 99

Milgrom M.: 1983, ApJ 270, 365

Milgrom M.: 1998, ApJ 496, L89

Milgrom M., Sanders R.H.: 2007 ApJ 658, 17

Moore B., Ghigna S., Governato F. et al.: 1999, ApJ 524, L19 
Naab, T., Burkert, A. 2003, ApJ 597, 893

Navarro, J.F., Steinmetz, M.: 2000, ApJ 528, 607

Nipoti, C., Londrillo, P., Ciotti, L., 2007, MNRAS 381, 104

Nipoti, C., Ciotti, L., Binney, J., Londrillo, P.: 2008, MNRAS 386, 2194

Noguchi M.: 1999, ApJ 514, 77

Sanders R.H., McGaugh S.: 2002, ARAA 40, 263

Schaye, J., Crain, R. A., Bower, R. G. et al.: 2015, MNRAS 446, 521

Sheth, K., Elmegreen, D. M., Elmegreen, B. G. et al.: 2008, ApJ 675, 1141

Silk, J., Mamon G.A.: 2012, Research in Astronomy and Astrophysics, Volume 12, Issue 8, pp. 917-946

Stott, J. P., Sobral, D., Smail, I. et al.: 2013, MNRAS 430, 1158

Swaters R.A., Sancisi R., van Albada T.S., van der Hulst J.M.: 2009 A\&A 493,871

Tacconi L., Genzel R., Neri R. et al.: 2010, Nature 463, 781

Tiret O., Combes F.: 2007, A\&A 464, 517

Tiret O., Combes F.: 2008a, A\&A 483, 719

Tiret O., Combes F.: 2008b ASPC 396,259

Toomre A.: 1977, in Evolution of Galaxies and Stellar Populations, Proceedings of a Conference at Yale University, p. 401

Vogelsberger, M., Genel, S., Springel, V. et al.: 2014, Nature 509, 177

Weinberg, M.D.: 1989, MNRAS 239, 549

Weinzirl T., Jogee S., Khochfar S. et al.: 2009, ApJ 696, 411

Wu, X., Zhao, H-S., Famaey, B. et al: 2007, ApJ 665, L101 